\title{
Antimicrobial Evaluation of Some Novel Aldimine Derivatives
}

\author{
H. V. SHAHARE ${ }^{1 *}$, L. P. KOTHARI ${ }^{1}$, S. M. BHAVSAR ${ }^{1}$ and R. J. BHOR \\ ${ }^{1}$ Dept. Phy. Chem., SNJB's SSDJ College of Pharmacy Chandwad, Nashik, \\ Maharashtra-423101, India \\ ${ }^{2}$ PRES's Pravaranagar College of Pharmacy Chincholi, Nashik, \\ Maharashtra- 422102, India \\ hvshahare@gmail.com
}

Received 27 July 2018 / Accepted 31 August 2018

\begin{abstract}
Some aldimine (or Schiff bases) derivatives have been synthesized by simple condensation of aromatic aldehydes with primary aromatic amines. Their structures have been elucidated by IR and ${ }^{1} \mathrm{H}$ NMR. The biological activity of these aldimines was examined against different type of microorganisms and they found to have considerable activity in comparison with the most commonly used antibiotics.
\end{abstract}

Keywords: Aldimines, Imine, Schiff bases, Antimicrobial activity

\section{Introduction}

The chemistry of heteropolar unsaturated functions are very well explored ${ }^{1,2}$. Aldimine (or Schiff bases); bimolecular condensation products of primary amines with aldehydes represent a valuable substrate in synthesis of a large number of industrial compounds via ring closure, cycloaddition, replacement reaction and etc. Similarly these compounds and their complexes have a variety of applications in biological, clinical and analytical fields ${ }^{3,4}$.

\section{Experimental}

All chemicals and solvent were purchased from Aldrich, ${ }^{1} \mathrm{H}$ NMR spectra were recorded with Brucker AC 300 spectrophotometer using $\mathrm{CDCl}_{3}$ and reported relative to TMS as an internal standard and IR spectra were recorded on Jasco FTIR 4100.

General scheme for preparation of aldimines

The aldimine were prepared (Scheme 1) by a modification of the reported methods ${ }^{5-8}$.<smiles>Nc1cccc(O)c1</smiles><smiles>O=Cc1ccc(P)cc1</smiles><smiles>Oc1cccc(N=Cc2ccc(Br)cc2)c1</smiles>

Figure 1. General scheme for synthesis 
A typical procedure for the synthesis of aldimines is as follows

A solution of 0.01 mole of the amine was slowly added to a solution of 0.01 mole of the aldehyde. The reaction mixture was stirred for 45 minutes at $40-50{ }^{\circ} \mathrm{C}$, the precipitate was cooled and collected by filtration. Further, washed several times with distilled water. The products were recrystallised from a mixture of water and ethanol (1:1) and dried in air. Details of their physical properties are given in Table 1.

\section{Results and Discussion}

The purity and structure of synthesized compounds were confirmed by thin layer chromatography, melting point, FTIR and ${ }^{1} \mathrm{H}$ NMR spectroscopy. IR spectra were recorded by using Jasco FTIR 4100 spectrophotometer. ${ }^{1} \mathrm{H}$ NMR spectra were recorded by using TMS as an internal standard. Melting points were determined in open capillary tube method and are uncorrected.

Table 1. Physical property of synthesized aldimine derivatives

\begin{tabular}{cccccc}
\hline Compd No. & $\mathrm{R}$ & $\begin{array}{c}\text { Mol. } \\
\text { Formula }\end{array}$ & $\begin{array}{c}\text { Melting } \\
\text { pint, }{ }^{\circ} \mathrm{C}\end{array}$ & $\begin{array}{c}\text { Percentage } \\
\text { yield, \% }\end{array}$ & $\mathrm{R}_{\mathrm{f}}$ value \\
\hline $\mathbf{S 1}$ & $4-\mathrm{Cl}$ & $\mathrm{C}_{13} \mathrm{H}_{10} \mathrm{NOCl}$ & $54-56$ & 69.04 & 0.62 \\
$\mathbf{S 2}$ & $4-\mathrm{OH}$ & $\mathrm{C}_{13} \mathrm{H}_{11} \mathrm{NO}_{2}$ & $48-51$ & 62.19 & 0.56 \\
$\mathbf{S 3}$ & $4-\mathrm{OCH}_{3}$ & $\mathrm{C}_{14} \mathrm{H}_{13} \mathrm{NO}_{2}$ & $62-64$ & 71.21 & 0.70 \\
$\mathbf{S 4}$ & $2,4,6-\left(\mathrm{OCH}_{3}\right)_{3}$ & $\mathrm{C}_{16} \mathrm{H}_{17} \mathrm{NO}_{4}$ & $124-127$ & 57.72 & 0.84 \\
$\mathbf{S 5}$ & $4-\mathrm{N}\left(\mathrm{CH}_{3}\right)_{2}$ & $\mathrm{C}_{15} \mathrm{H}_{16} \mathrm{~N}_{2} \mathrm{O}$ & $70-72$ & 66.40 & 0.69 \\
$\mathbf{S 6}$ & $4-\mathrm{NO}_{2}$ & $\mathrm{C}_{15} \mathrm{H}_{16} \mathrm{~N}_{2} \mathrm{O}$ & $66-68$ & 70.08 & 0.82 \\
S7 & $4-\mathrm{F}$ & $\mathrm{C}_{15} \mathrm{H}_{16} \mathrm{~N}_{2} \mathrm{O}$ & $64-65$ & 73.87 & 0.76 \\
\hline
\end{tabular}

Spectral Data: IR spectra and ${ }^{I} H$ NMR

The spectra shows no absorption at about $1700 \mathrm{~cm}^{-1}$ which indicates the absence of free carbonyl group (Table 2). The strong absorption in the $1620 \mathrm{~cm}^{-1}$ region is assigned to the azomethine group present in the Schiff bases. The ${ }^{1} \mathrm{H}$ NMR spectra shows the common signal which appears at 10.2 and $6.9-8.0 \mathrm{ppm}$, in all compounds was assigned to azomethine and aromatic protons respectively (Table 3 ).

Table 2. IR spectral data of aldimine derivatives

\begin{tabular}{|c|c|}
\hline Compd. No. & IR Data, $\mathrm{cm}^{-1}$ \\
\hline S1 & $\begin{array}{l}1608(\mathrm{C}=\mathrm{N}), 1320(\mathrm{C}-\mathrm{N}), 852(\mathrm{Cl}), 760(\mathrm{Ar}-\mathrm{CH}), 3422(\mathrm{OH}), 3046 \text { (Alkene } \\
\mathrm{C}-\mathrm{H})\end{array}$ \\
\hline S2 & $1594(\mathrm{C}=\mathrm{N}), 1365(\mathrm{C}-\mathrm{N}), 696(\mathrm{Ar}-\mathrm{CH}), 3416(\mathrm{OH}), 3030($ Alkene C-H) \\
\hline S3 & $\begin{array}{l}1597(\mathrm{C}=\mathrm{N}), 1282(\mathrm{C}-\mathrm{N}), 814(\mathrm{Ar}-\mathrm{CH}), 3408(\mathrm{OH}), 3055(\text { Alkene } \mathrm{C}-\mathrm{H}) \text {, } \\
1462\left(\mathrm{OCH}_{3}\right)\end{array}$ \\
\hline S4 & $\begin{array}{l}1602(\mathrm{C}=\mathrm{N}), 1312(\mathrm{C}-\mathrm{N}), 689(\mathrm{Ar}-\mathrm{CH}), 3419(\mathrm{OH}), 3024(\text { Alkene } \mathrm{C}-\mathrm{H}), \\
1440\left(\mathrm{OCH}_{3}\right)\end{array}$ \\
\hline S5 & $\begin{array}{l}1614(\mathrm{C}=\mathrm{N}), 1216(\mathrm{C}-\mathrm{N}), 3464(\mathrm{OH}), 1355\left(\mathrm{CH}_{3}\right), 754(\mathrm{Ar}-\mathrm{CH}), 3114 \\
(\text { Alkene C-H) }\end{array}$ \\
\hline S6 & $\begin{array}{l}1612(\mathrm{C}=\mathrm{N}), 1282(\mathrm{C}-\mathrm{N}), 698(\mathrm{Ar}-\mathrm{CH}), 3402(\mathrm{OH}), 1474\left(\mathrm{NO}_{2}\right), 3121 \\
(\text { Alkene C-H) }\end{array}$ \\
\hline S7 & $\begin{array}{l}1619(\mathrm{C}=\mathrm{N}), 1374(\mathrm{C}-\mathrm{N}), 952(\mathrm{~F}), 784(\mathrm{Ar}-\mathrm{CH}), 3326(\mathrm{OH}), 3178 \text { (Alkene } \\
\mathrm{C}-\mathrm{H})\end{array}$ \\
\hline
\end{tabular}


Table 3. ${ }^{1} \mathrm{H}$ NMR data of aldimine derivatives

\begin{tabular}{cl}
\hline Compd. No. & \multicolumn{1}{c}{ NMR Data $(\delta \mathrm{ppm})$} \\
\hline S5 & 6.955-8.012 $(\mathrm{Ar}, \mathrm{m}, 7 \mathrm{H}), 10.254($ Azomethine, s, 1H), $9.360(\mathrm{OH}, \mathrm{s}, 1 \mathrm{H})$, \\
& $2.609\left(\mathrm{CH}_{3}, \mathrm{~s}, \mathrm{6H}\right)$ \\
S7 & 6.9840-8.236 $(\mathrm{Ar}, \mathrm{m}, 7 \mathrm{H}), 10.271$ (Azomethine, s, 1H), 9.397 (OH, s, 1H), \\
\hline
\end{tabular}

Biological assay

Antibacterial activity of the aldimines has been carried out against some bacteria such as $S$. aureus and E. coli. using nutrient agar medium by well diffusion method. The concentration of samples ranged from $2-4 \mathrm{mg} / \mathrm{mL}$ in pure methanol ${ }^{9}$. The results are expressed in MIC (minimal inhibitory concentration) solvent blanks were run against each test organism in all assays; the results of antimicrobial activity is shown in Table 4.

Table 4. Antimicrobial activity of aldimine derivatives

\begin{tabular}{cccccc}
\hline Compound & Substituent's & \multicolumn{2}{c}{ E. coli } & \multicolumn{2}{c}{ Staph. Aureus } \\
\cline { 3 - 6 } No. & $(\mathrm{R})$ & 0.1 & 0.01 & 0.1 & 0.01 \\
\hline S1 & $4-\mathrm{Cl}$ & 3.1 & 1.7 & 2.9 & 1.7 \\
S2 & $4-\mathrm{OH}$ & 4.2 & 2.3 & 3.6 & 2.2 \\
$\mathbf{S 3}$ & $4-\mathrm{OCH}_{3}$ & 3.7 & 1.6 & 3.1 & 1.4 \\
$\mathbf{S 4}$ & $2,4,6-\left(\mathrm{OCH}_{3}\right)_{3}$ & 4.1 & 2.5 & 3.9 & 2.1 \\
S5 & $4-\mathrm{N}^{2}\left(\mathrm{CH}_{3}\right)_{2}$ & 5.8 & 3.4 & 5.2 & 3.1 \\
S6 & $4-\mathrm{NO}_{2}$ & 3.9 & 2.7 & 3.2 & 2.4 \\
S7 & 4-F & 5.7 & 3.3 & 5.0 & 3.2 \\
Standard & Ampicillin & 7.2 & 4.6 & 6.9 & 4.3 \\
Solvent & DMF & - & - & - & - \\
\hline
\end{tabular}

\section{Conclusion}

Some new aldimine derivatives (Schiff base) were synthesized successfully by simple and one step method in a good yield. Among these, compound S5 and S7 shows very potential antibacterial activity and shows excellent scope for further development as commercial antimicrobial agents.

\section{References}

1. Comprehensive Organic Chemistry, Synthesis and Reactions of Org. Compounds, Ed., Sir Derek Barton, Vol. 2 part 8 p. 383, London.

2. $\quad$ Koll A, Int J Mol Sci., 2003, 4(7), 434-444; DOI:10.3390/i4070434

3. Brown A D and Colvin E V, Tetrehedron Lett., 1991, 32(38), 5187-5190; DOI:10.1016/S0040-4039(00)93462-2

4. Burtnett D A, Gallucci J C and Hart D J, J Org Chem., 1985, 50(25), 5120-5123; DOI:10.1021/jo00225a028

5. Marzilli L G and Halpern J, J Am Chem Soc., 1971, 93(6), 1374-1378; DOI:10.1021/ja00735a010

6. Bigelow L A and Gatough H, Org Synth Coll., 1967, I, 80.

7. Huang J M, Zhang J F, Dong Y and Gong W, J Org Chem., 2011, 76(9), 35113514; DOI:10.1021/jo102455q

8. Morales S, Fernando G G, García Ruano J L and Belén Cid M, J Am Chem Soc., 2014, 136(3), 1082-1089; DOI:10.1021/ja4111418

9. Zainab H, Emad Y, Ahmed A and Altaie A, Org Med Chem Lett., 2014, 4, 1, DOI:10.1186/2191-2858-4-1 